\title{
10 General conclusions
}

In the previous chapters, I analyzed the phenomenon of verbal degree gradation. The thesis has both a semantic and a comparative perspective due to its comparisons of degree gradation in German, Russian and French. It builds on the work of Bolinger (1972); Ropertz (2001) and Löbner (2012b) but extends these works by taking more data and, more importantly, a wider range of languages into account. Furthermore, the current thesis contains different case studies that are inspired by the work of Ropertz (2001) but go into greater detail. Verbal degree gradation is a less studied but central semantic topic, as it raises questions concerning the notion of compositionality, telicity and the interaction between lexical semantics and conceptual knowledge. In this last chapter, I like to summarize the central results of the thesis and to indicate open questions which arise from the analyses presented in the various chapters.

As a first general result of the thesis, I demonstrated that the same picture of verbal degree gradation can be observed in the three abovementioned languages, although the languages do, in fact, show some differences in the formal realization of degree gradation (using a ' $\mathrm{d}$ '-adverbial in German and Russian but a 'd/e'-adverbial in French) or grammatical aspect.

The starting point of the analysis is the observation, which goes back to Bolinger (1972), that verb gradation is not a uniform process; rather it can be separated into verbal degree gradation and verbal extent gradation. Degree gradation involves specifying a degree on a scale associated with the verbal predication, whereas extent gradation entails specifying a gradable property of the event such as its temporal duration or frequency. In chapter 4, I demonstrated that both types of verbal degree gradation are related to different syntactic configurations. Degree gradation is expressed by a nuclear adverbial which modifies the predicate of the sentence. Extent gradation is expressed by core adverbials which modify the event description consisting of the predicate and its arguments. In languages like 
French that use the same expression for extent and degree gradation the adverbial is syntactically ambiguous. In German and Russian on the other hand, which use different expressions for extent and degree gradation, no such syntactic ambiguity exists.

One question that is not addressed in the thesis is how verbal constructions like the resultative construction or a verbal comparison construction fit into the presented syntactic analysis. In an example like (1), sehr is used for verbal degree gradation which would require nach Blumen riechen 'smell of flowers' to be a nuclear juncture. Independent evidence for this assumption is required to maintain the analysis. If such evidence can be found, verbal degree gradation can be used as an indicator for the syntactic layer of a certain construction.

\section{Die Katze riecht sehr nach Blumen. the cat smells very of flowers 'The cat smells very much of flowers.'}

The comparison of verbal degree gradation with adjectival degree gradation revealed two essential differences. First, whereas gradable adjectives encode a measure function and therefore lexically express a scalar predication, this is different for verbs. There are some clear cases of lexically scalar verbs, such as change of state verbs and some stative dimensional verbs such as cost or weigh. But most gradable verbs are not lexically scalar, which means that they do not encode a scale in their lexical semantics. Non-scalarity of these verbs is demonstrated by the fact that in most of their uses they do not express a comparison between degrees, whereas scalar verbs do. Wachsen 'grow' (2) expresses such a comparison in each context of use as it means 'become taller than before.' But verbs such as bluten 'bleed,' stinken 'stink' or lieben 'love' do not express a comparison in (3). In (3a), it is not expressed that the wound bled more than it normally does or than another wound has bled. Similarly in (b), it is not said that the dog smells more than normally or some other dog or dogs in general or more unpleasant than usual and in (c) it is not expressed that the boy loves his mother more than someone else or than before. Hence, the ungraded sentences in (3) do not express a comparison and therefore cannot be conceived as being scalar. 
(2) Das Kind ist gewachsen.

the child is grown

'The child has grown.'

(3) a. Die Wunde hat geblutet.

the wound has bled

'The wound bled.'

b. Der Hund stinkt.

the dog stinks

'The dog stinks.'

c. Der funge liebt seine Mutter.

the boy loves his mother

'The boy loves his mother.'

Verbal degree gradation is regular for semantic classes of verbs such as verbs of smell/sound/light emission, verbs of substance emission, experiencer verbs or action verbs. In each case, the gradation scale is the same for the verbs of the respective semantic class. Most classes of verbs also provide a single scale for verbal degree gradation. Only gradable action verbs seem to license two different gradation scales; one related to the manner component of the verb, the other measuring the effect of the action described by the verb. The gradation scale is constrained by the lexical semantics of the verb. I argued in chapter 5 that if the scale is not lexically encoded in the verb it is retrieved from the conceptual knowledge associated with it. But this process of retrieval (or attribute activation) is not arbitrary, since only meaning components lexically specified in the verb give access to conceptual knowledge. One crucial question, not raised in the thesis, is why these meaning components give, at least in most cases, only access to a single scale. A more detailed investigation of this topic is left open for the future.

The semantic representation of bluten 'bleed' is given in (4). It consists of four conjuncts which specify the event, the emitter and the implicit emittee of the emission. Only the implicit emittee argument is lexically specified as being blood and therefore it is only this argument that gives access to conceptual knowledge. The conceptual knowledge here is encyclopedic information about the object 'blood.' This includes, among other things, the knowledge that blood comes in a certain quantity and therefore licenses the retrieving of the quantity attribute (cf. Fleischhauer 2015 for a frame- 
based representation of verbs of substance emission and the conceptual knowledge given excess to by these verbs).

$$
\text { 【bluten } \rrbracket=\lambda \mathrm{x} \lambda \mathrm{v}(\operatorname{emit}(\mathrm{v}) \wedge \operatorname{EMITTER}(\mathrm{v})=\mathrm{x} \wedge \operatorname{THEME}(\mathrm{v})=\mathrm{s} \wedge \operatorname{blood}(\mathrm{s}))
$$

As the emitter argument is not lexically specified, it does not give access (at least at the level of the predicate) to conceptual knowledge. Therefore sehr cannot modify a gradable property of the emitter but only of the emittee.

The fact that most gradable verbs do not lexicalize a scale gives rise to the assumption that we can enrich the lexical meaning of the verb by relying on conceptual knowledge. As argued above, this process is not unconstrained and therefore the enriched concept cannot include just any arbitrary attribute but rather only attributes licensed by a meaning component specified in the lexical meaning of the verb. A suitable format for representing this process of attribute activation is frame theory as described by Löbner (2014) as well as Petersen (2007). The advantage of the frame approach is that it easily allows the combining of semantic representations of verbs with the conceptual knowledge associated with one of the meaning components. Hence, there is no strict demarcation of lexical and conceptual knowledge in this approach. A next step in the semantic analysis would be an explicit frame analysis of verbal degree gradation (see Fleischhauer et al. 2014 for a first step towards such an analysis).

The second crucial difference between verbal degree gradation and adjectival degree gradation is the fact that degree gradation of verbs is a subcompositional phenomenon. This claim goes back to Löbner (2012b) who states that a morphosyntactic construction is subcompositional if it requires different rules of semantic composition. This is the case for verbal degree gradation, as the different compositional patterns summarized in chapter 9 cannot be reduced to a single one. Rather, each semantic class of verbs requires its own rule of composition. This is due to the fact that the respective gradation scale is differently linked to the eventuality for each semantic class of verbs. Moreover, different classes of verbs are related to different types of scales, some measuring intensity, others a divergence. A different picture emerges for adjectival degree gradation, as the different cases can be accounted for by a single rule of semantic composition. The fact that verbal degree gradation is a subcompositional phenomenon shows that the assumption of the homomorphism of semantic and syntac- 
tic composition does not hold. This means that the same rule of semantic composition does not apply for every complex expression of a particular syntactic composition. It is an open question as to which further constructions qualify as subcompositional, but adverbial modification in general seems to have a subcompositional flavor. In (5a), the adverbially used adjective schnell 'fast, quick' expresses that it took a short time till Peter responded but it is the manner of the responding that is fast, meaning Peter is speaking fast. In (b), schnell indicates the speed of running and not that it took Peter a short time till he ran. ${ }^{1}$
a. Peter antwortete schnell.
Peter responded quick
'Peter responded quickly.'
b. Peter lief schnell.
Peter ran fast
'Peter ran fast.'

Note that this is a different analysis to the one proposed by, for example, Dowty (1979) for the different readings of English almost with activity predicates and accomplishment predicates (see the discussion in chapter 3.2.1). Dowty is merely speaking of a scope ambiguity but does not account for the different readings by different rules of composition. In the tradition of generative semantics, the different readings of almost have been taken as an argument in favor of lexical decomposition (e.g. Morgan 1969). The same is true for the ambiguity of again. The subcompositionality analysis does not simply assume that a scope ambiguity arises due to different decompositional structures but that the different interpretations of the sentences in (5) as well as of verbal degree gradation arises due to different rules of composition. This has not, as far as I know, been claimed so far in the discussion of the ambiguity of almost and again.

Different compositional patterns of verbal degree gradation have been demonstrated in detail for three semantic classes of verbs: change of state verbs, verbs of emission and experiencer verbs. It has also been demonstrated - in less detail - for some other classes of verbs in chapter 9; namely,

The reading that it took Peter a short while till he started running is possible but without further context, the reading that schnell indicates the speed of running is preferred. 
verbs of comparison, erratic verbs, verbs of marked behavior and action verbs. A large class of verbs neglected in this thesis is communication verbs. Examples are the verbs versprechen 'promise' or prahlen 'boast.' This class is rather heterogeneous with respect to argument realization as well as the lexical semantics of the verbs and it is connected to the expression of speech acts. Hence, in some of their uses they take sentential complements: ample of examples of these verbs, showing that it is a very productive verb class regarding verbal degree gradation, are contained in the German database.

A deeper analysis of German erratic verbs would also be of interest, as in this case the scale is introduced by a verbal particle. Deriving erratic verbs is rather productive in German and would provide an interesting case study on the interaction between degree gradation and verbal prefxiation. This would be of relevance as the scale induced by the prefix blocks the access to a scale associated with the verb (as discussed in chapters 7 and 9).

To get the full picture of verbal degree gradation, a broader corpus-based study would be required with the aim of showing which verbs actually license degree gradation and which not. A first step towards such an analysis has already been undertaken by Sebastian Löbner and resulted in a database containing several thousand examples of gradable verbs. ${ }^{2}$ However, to gain a broader view on verbal degree gradation would also require a comparative (corpus-based) study of different intensifiers, as the thesis only focusses on sehr and its correspondents in other languages. Other intensifiers might show different restrictions from sehr and therefore reveal interesting insights into verbal degree gradation from a more general perspective.

A central issue in the analysis of verbal degree gradation has been the interaction with grammatical as well as lexical aspect. It emerged that grammatical aspect affects the interpretation of verbal degree gradation but only in case of (atelic) change of state verbs and verbs of substance emission. In case of a perfective interpretation, the intensifier indicates the total amount of change, or the total quantity of emitted substance. Progressive aspect restricts the denotation to a subevent, and the intensifier specifies the change

The database contains examples collected in the project 'Verb gradation' headed by Sebastian Löbner and financed by the Deutsche Forschungsgemeinschaft 'German Research Foundation' (DFG grand LO 454/1). 
or emitted quantity at a stage of the event. This phenomenon has been called 'event-dependent degree gradation,' and it arises if the degree on the scale is coupled with the progression of the event such that the degree increases if the event unfolds. An analysis in terms of a homomorphic mapping between the ordered set of degrees and the part structure of the event has been presented. Change of state verbs as well as verbs of substance emission express an incremental change on their respective scales. In case of atelic change of state verbs, degree gradation by sehr results in a telic predication as the intensifier indicates a lower bound that has to be reached in the event. Regarding verbs of substance emission degree gradation does not have an effect on telicity, although the implicit emittee argument is really an implicit incremental theme argument. This has been demonstrated by the fact that quantization of the argument results in a telic predication. Examples like those in (6) illustrate that sehr is not able to quantize the implicit incremental theme argument and therefore the graded predication remains atelic.

(6) a. \#Die Wunde hat sehr geblutet in zehn Minuten. the wound has very bled in ten minutes 'The wound bled a lot in ten minutes.'

b. \#Die Wunde hat sehr geeitert in zehn Minuten. the wound has very festered in ten minutes 'The wound festered a lot in ten minutes.'

c. \#Es hat sehr geregnet in zehn Minuten. it has very rained in ten minutes 'It rained a lot in ten minutes.'

A conclusive explanation of why sehr is not able to render verbs of substance emission telic is still missing. Further work on degree gradation and implicit incremental theme arguments is required as it sheds light onto the central notion of telicity.

A further connection between degree gradation and telicity exists in the case of graded accomplishment predicates. Since some accomplishment change of state predications like stabilisieren 'stabilize' or normalisieren 'normalize' are gradable, a telos cannot necessarily be equated with a maximum scale value. Rather it has been demonstrated that two types of telos need to be distinguished: a maximum telos which is equal to the maxi- 
mum scale value and a standard telos which represents the onset of an extended result scale. The cross-linguistic discussion revealed that accomplishment change of state predicates are gradable if they are related to a standard telos but that they reject degree gradation by sehr, očen' or beaucoup if they are only related to a maximum telos. It would be interesting to see whether these observations easily connect with the phenomenon of so-called 'non-culminating accomplishments' (see Koenig \& Muansuwan 2000; Chief 2007; Koenig \& Chief 2008; Tatevosov 2008 among others). Examples from Mandarin Chinese are shown in (7).
a. Zhangsan sha le Lisi liang ci, Lisi dou mei si. Zhangsan kill PF Lisi two time Lisi all not die 'Zhangsan killed Lisi twice, but Lisi didn't die. (intended read- ing)'
b. wo kai le men (danshi men mai kai).
I open PF door but door not open
'I opened the door, (but the door was not opened).'
(Chief, 2007, 32)

The verbs sha 'kill' and kai 'open' are telic but nevertheless the attainment of a maximal degree can be negated without contradiction. A question would be whether this incompleteness effect, as Chief calls it, can also be accounted for by a distinction between standard and maximum telos. This would probably allow unifying different telicity related phenomena under a single analysis and to find parallels between different and seemingly unrelated phenomena.

A topic connected to verb gradation is verbal scalarity. Three different sources of verbal scales have been mentioned in chapter 5 . They can either be lexicalized by a verb, retrieved from conceptual knowledge or introduced by a morphosyntactic construction. The focus of the thesis has been on the first two options: the third one has only been investigated superficially. It was mentioned that the resultative construction and certain types of verbal comparison constructions introduce scales but also verbal prefixes and particles.

Prefixes and particles are either able to introduce a new scale like in the Polish examples in (8) or to modify a scale associated with the verb (9). Whereas in (8b) the prefix shifts the stative verb of smell emission 
towards an eventive change of state predication, in (9) the prefix introduces an endpoint to a non-lexicalized scale. ${ }^{3}$
a. Jan bardzo śmierdziat.
Jan very stank
'Jan stank very much.'
b. Jan bardzo za-śmierdziat.
Jan very ZA-stank
'Jan began to stink very much.'

(9) Das Schwein war in zehn Minuten ausgeblutet.

the pig was in ten minutes out.bled

'The pig bled out in ten minutes.'

A typology of scalar constructions, i.e. morphosyntactic constructions that introduce a verbal scale, is still missing and it is an open question which types of further constructions count as scalar. An additional question is how scale components are distributed within a sentence. (9) shows an example in which the verbal particle adds information to the scalar predication, the incremental change on the quantity scale is bounded. Another case of distributed scalar information has been seen with regard to scalar underspecification discussed in chapter 6. In examples like those in (10) the scale parameters (dimension, set of degree and linear ordering relation) is specified by the scale-denoting nouns. It would be interesting to broaden the perspective and to investigate how scales and their components are morphosyntactically encoded in different languages.
a. Der Druck steigt.
the pressure rises
'The pressure is rising.'
b. Der Preis steigt.
the price rises
'The price is rising.'
c. Die Temperatur steigt.
the temperature rises
'The temperature is rising.'

3 See Kagan (2015) for a recent scalar analysis of verbal prefixes in Russian. 
The thesis investigates an empirical domain which has not received much attention so far. It has been shown that verbal degree gradation is not a marginal phenomenon as many verbs are gradable. It has been shown that the topic of verbal degree gradation is related to other topics independently discussed in syntax and semantics as, for example, scalarity, telicity and compositionality. But it also followed that there are hugh differences between adjectival and verbal degree gradation. The current work is just a first step into the analysis of verbal degree gradation but it indicates many open questions for further research. A central issue is deeper lexical decomposition which is required by verbal degree gradation and probably by adverbial modification in general. Attempts towards such a deeper lexical decomposition have been undertaken but many open questions still need to be investigated. 